\title{
The Plastid Genome of Eutreptiella Provides a Window into the Process of Secondary Endosymbiosis of Plastid in Euglenids
}

\author{
Štěpánka Hrdá ${ }^{1}$, Jan Fousek ${ }^{2}$, Jana Szabová ${ }^{1}$, Vladimír Hampl V ${ }^{1 *}$, Čestmír Vlček ${ }^{2 *}$ \\ 1 Charles University in Prague, Faculty of Science, Department of Parasitology, Prague, Czech Republic, $\mathbf{2}$ Institute of Molecular Genetics, Academy of Sciences of the \\ Czech Republic, Prague, Czech Republic
}

\begin{abstract}
Euglenids are a group of protists that comprises species with diverse feeding modes. One distinct and diversified clade of euglenids is photoautotrophic, and its members bear green secondary plastids. In this paper we present the plastid genome of the euglenid Eutreptiella, which we assembled from 454 sequencing of Eutreptiella gDNA. Comparison of this genome and the only other available plastid genomes of photosynthetic euglenid, Euglena gracilis, revealed that they contain a virtually identical set of 57 protein coding genes, 24 genes fewer than the genome of Pyramimonas parkeae, the closest extant algal relative of the euglenid plastid. Searching within the transcriptomes of Euglena and Eutreptiella showed that 6 of the missing genes were transferred to the nucleus of the euglenid host while 18 have been probably lost completely. Euglena and Eutreptiella represent the deepest bifurcation in the photosynthetic clade, and therefore all these gene transfers and losses must have happened before the last common ancestor of all known photosynthetic euglenids. After the split of Euglena and Eutreptiella only one additional gene loss took place. The conservation of gene content in the two lineages of euglenids is in contrast to the variability of gene order and intron counts, which diversified dramatically. Our results show that the early secondary plastid of euglenids was much more susceptible to gene losses and endosymbiotic gene transfers than the established plastid, which is surprisingly resistant to changes in gene content.
\end{abstract}

Citation: Hrdá Š, Fousek J, Szabová J, Hampl V V, Vlček Č (2012) The Plastid Genome of Eutreptiella Provides a Window into the Process of Secondary Endosymbiosis of Plastid in Euglenids. PLoS ONE 7(3): e33746. doi:10.1371/journal.pone.0033746

Editor: Jonathan H. Badger, J. Craig Venter Institute, United States of America

Received November 23, 2011; Accepted February 16, 2012; Published March 20, 2012

Copyright: (c) 2012 Hrdá et al. This is an open-access article distributed under the terms of the Creative Commons Attribution License, which permits unrestricted use, distribution, and reproduction in any medium, provided the original author and source are credited.

Funding: This work was supported by Ministry of Education, Youth and Sport of the Czech Republic (project MSM0021620828), by Czech Science Foundation P506/11/1320 to VH, and by the Grant Agency of the Charles University 63409 to JS. The funders had no role in study design, data collection and analysis, decision to publish, or preparation of the manuscript.

Competing Interests: The authors have declared that no competing interests exist.

*E-mail: vlada@natur.cuni.cz (VH); vlcek@img.cas.cz(ČV)

\section{Introduction}

Euglenids are a relatively large group of protists that contains species with different types of feeding strategies: some euglenid species (e.g. Rhabdomonas) are osmotrophic and feed by pinocytosis; others developed phagotrophic apparatuses for catching bacteria (e.g. Entosiphon) or even eukaryotes (e.g. Peranema) [1,2]. One large clade of euglenids is photoautotrophic and its members bear green secondary plastids (e.g. Euglena gracilis). The plastid has been subsequently and independently lost in several branches within this clade (Euglena longa, prev. Astasia longa, Euglena quartana, prev. Khawkinea quartana, Euglena hyalina, Euglena viridis hyalina and Phacus ocellatus, prev. Hylophacus ocellatus) [3-5]. The phototrophic euglenids and their secondary heterotrophic descendents are classified as class Euglenophyceae [4]. Complete plastid genome sequences are known so far for only two closely related euglenid species, Euglena gracilis [6] and Euglena longa [7].

The fact that plastids are present in a single clade of euglenids favors a hypothesis that the ancestor of this clade acquired the plastid by engulfing a green alga $[6,8]$. Our current knowledge on the phylogeny of euglenids implies that this endosymbiotic event happened after the split of Peranema but before the split of Eutreptiella and Eutreptia, the basal lineages of the phototrophic clade [9]. This "plastid late" hypothesis is further indirectly supported by the fact that the autotrophic clade is derived from within the eukaryovorous euglenids; eukaryovory is regarded as the derived feeding mode in euglenids and at the same time it is a useful predisposition facilitating the engulfment of green algae [10]. The plastid of Euglena gracilis can be completely lost after bleaching with many enviromental and chemical agents without effect on cell viability, and this fact is also used as an argument for a relatively recent acquisition of the plastid, which has not yet been recruited for cellular functions other than photosynthesis [11]. Recent study of introns in the plastid targeting presequences also agrees with the plastid-late hypothesis [12]. An alternative but currently less-accepted plastid-early hypothesis postulates that the euglenid plastid was acquired early in the evolution of euglenids, or even in the common ancestor of euglenids and kinetoplastids (e.g. Trypanosoma), their nearest sister group $[13,14]$. The presence of genes of red algal origin in the photosynthetic Euglena as well as in the heterotrophic Peranema suggests that the lineage of euglenids might have experienced a cryptic red algal plastid endosymbiosis before the current green algal plastid was established [15].

Analyses of 70 plastidial genes and conservation of gene order on the plastid genome has pointed to Pyramimonas (Pyramimonadales, Prasinophyceae) as the closest extant relative of the euglenid plastid [7]. Pyramimonas comprises marine flagellates, suggesting that the endosymbiotic event happened in the marine environ- 
ment. Although the majority of euglenids live in freshwater, the basal lineage of the autotrophic clade contains the marine species Eutreptia and Eutreptiella, corroborating the hypothesis of a marine origin of photosynthetic euglenids [4,5]. The comparative analysis of the gene content between the plastid genome of Pyramimonas parkeae, which encodes 110 conserved genes (81 protein and 29 RNA species) [16], and Euglena gracilis, which comprises 88 conserved genes (58 protein and 30 RNA species) [6], has revealed a substantial loss of genes (for example all genes of NADHplastoquinone oxidoreductase of the plastidial respiratory chain) happening from the common ancestor of $P$. parkeae and $E$. gracilis to extant $E$. gracilis. This reduction of gene repertoire is explained as a consequence of secondary endosymbiosis, although comparable gene losses took place in the prasinophyte lineages leading to Pycnococcus and to the coccoid microalgae Ostreococcus and Monomastix [16]. Further gene loss in euglenids accompanying the loss of photosynthetic activity has been observed in the closely related but non-photosynthetic Euglena longa, which has maintained 56 conserved genes (26 protein and 30 RNA species) [7]. Despite the reduction of coding capacity of the Euglena plastid in comparison to that of $P$. parkeae, the size of the E. gracilis genome increased (143.2 vs. $101.6 \mathrm{~kb}$ in $P$. parkeae). The increase in the genome size should mainly be ascribed to the expansion of selfsplicing introns. While $P$. parkeae features a single group II intron, the genome of the E. gracilis plastid contains 160 group II and group III introns (15 of which formed twintrons), which is by far the most of all known organellar genomes [17,18]. There are indications that the expansion of introns may be a feature specific to $E$. gracilis and its relatives [17,18]; however, no other plastid genome of euglenids has been completely sequenced, which would be necessary to enable comprehensive comparisons.

Here we report the complete genome sequence of Eutreptiella gymnastica, a member of the basal lineage of the photosynthetic clade, and phylogenetically most distant from Euglena gracilis - the common ancestor of E. gracilis and E. gymnastica was the common ancestor of all currently known members of the photosynthetic lineage $[1,19,20]$. Comparative analysis of the gene content of euglenid plastids allows relatively precisely tracing the events of gene transfers and gene losses accompanying this particular case of secondary endosymbiosis. The vast differences in intron density suggest that the expansion of introns has happened specifically in the lineage leading to $E$. gracilis.

\section{Results and Discussion}

The complete size of the circular chloroplast DNA of Eutreptiella gymnastica is $67622 \mathrm{bp}$. An overview of the general features of this genome and its closest relatives is given in Table 1 . The genome sequence is numbered from the first nucleotide after the second 23S RNA gene (see a physical map of chloroplast DNA - Figure 1). The organization of the genome resembles those of higher plants and algae (including Pyramimonas parkeae) with a large single copy region (LSC), a small single copy region ( $\mathrm{SSC}$ ) and two inverted repeats (IR). Simplified maps of plastid genomes of Eutreptiella gymnastica, Euglena gracilis, Euglena longa, and Pyramimonas parkeae are illustrated in Figure 2 for comparison.

As is apparent from the genome map (Figure 1), the SSC region is reduced (to $1055 \mathrm{bp}$ ), containing only one ORF of unknown function (orf248). This is not surprising, because E. gymnastica (like E. gracilis) has lost most genes usually found in the SSC region (NADH dehydrogenase complex and a few others). Two of them (rpl32 and psaC) are relocated to other sites. The large single copy region (47 $528 \mathrm{bp}$ ) contains most genes for proteins and tRNAs.
Two regions resembling inverted repeats (IR, $6304 \mathrm{bp)} \mathrm{contain}$ one 16S rRNA gene (1463 bp), one 23S rRNA gene (2999 bp), and a 1726-bp-long sequence with unknown function that contains 2 tandem repeats - VNTR $(3 \times 11$ bp and $3,4 \times 33 \mathrm{bp})$. Between the IR copies, the 23S rRNA genes differ in three bases, while all other sequences are identical. The IR copy on the plus strand further contains an insertion of a block of genes (tRNA-Ala, tRNA-Cys, rps2, atpI, atpH, atpF, atpA, and orf372), and the IR copy on the minus strand contains the insertion of tRNA-Ile. The gene cluster of $\operatorname{rps} 2$, atpI, atpH, atpF, and atpA found within the IR is one of the ancestral gene clusters conserved in streptophyte and prasinophyte plastid genomes, but it is usually located in the LSC region [16]. The tRNA-Ala and tRNA-Ile genes are present also in the IR of $P$. parkeae.

The inverted repeats do not contain 5S RNA, and in fact Eutreptiella lacks it completely. Absence of 5S RNA was also recorded in the plastid genome of Pyramimonas parkeae and Pycnococcus provasolii, but the possibility exists that its sequence was unrecognized [16]. Interestingly, transcriptional analysis of the E. gracilis plastid chromosome showed that, although the genes for 5S, 23S and 16S RNA make one operon [6], the abundance of 5S RNA is much lower than the abundance of 23S and 16S RNA [21]. If 5S RNA is present but remains unrecognized in the plastid genome of Eutreptiella, it probably is not localized within the RNA operon, as the 16S RNA gene is very closely followed by neighboring genes. The symmetrical arrangement of tandem repeats in the non-coding part of the IRs suggests that this region may function as the origin of replication. According to the classical model [22], which has recently been challenged [23], the replication of plant and some green algal plastid genomes starts simultaneously from both IRs, and expands unidirectionally towards the SC region, forming two D-loop structures. After it passes the initiation site of the opposing D-loop, the two D-loops fuse to form Cairn-type bidirectional forks that move away from each other and meet approximately 180 degrees from the starting point. E. gracilis and E. longa plastid genomes lack IRs (Figure 2) and, so far, no model of their replication has been proposed. The origin of replication in the plastid genome of $E$. gracilis has been localized into the region of tandem repeats approximately $6 \mathrm{~kb}$ upstream from the extra 16S rRNA gene (Figure 2) [6,24,25]. From this site, the replication probably proceeds in both directions [6]. Because most genes are coded on the leading strand of replication, these genomes have a strikingly non-random distribution of genes. Starting from the ORI site, in one half of the circle, most genes are coded by the plus strand, and in the other half on the minus strand [6,26]. A similar situation is in Eutreptiella, but the switch of the coding strands is situated approximately $2 / 3$ of the way through the circle (Figure 1 and 2).

The size of the E. gymnastica plastid genome is less than half of that of E. gracilis, though the number of conserved genes in both species is not very different (Table 1). The difference in the genome size is caused by different numbers of self-splicing introns. The genome of E. gracilis plastid contains 160 group II and group III introns, which is by far the most of all known organellar genomes [6,17]. The plastid genome of Eutreptiella apparently contains only two putative introns, and in this respect it resembles the plastid genome of Pyramimonas parkeae, which contains only one [16]. We have not found any sequential, structural or positional homology either between the introns of Eutreptiella and Pyramimonas or between the introns of Eutreptiella and Euglena gracilis. The first putative intron of Eutreptiella (1480 bp) is located in the psaA gene. This intron apparently contains an orf386 (1158 bp) that shows very weak homology to reverse transcriptases. The homology is so weak that it was revealed only after iteration in PSI-BLAST. As 
Table 1. General features of euglenid and Pyramimonas cpDNA.

\begin{tabular}{|c|c|c|c|c|}
\hline Feature & Eutreptiella gymnastica & Pyramimonas parkae & Euglena gracilis & Euglena longa \\
\hline Genome size: & 67622 & 101605 & 143171 & 73345 \\
\hline GC percentage: & 34,32 & 34,7 & 26,13 & 22,41 \\
\hline Gene-unique loci: & 91 & 123 & 96 & 76 \\
\hline Unique rRNA (count/bases): & 2/8924 & 2/9086 & $3 / 15057$ & $3 / 15617$ \\
\hline Unique tRNA (count/bases): & $26 / 1959$ & $27 / 2393$ & $27 / 2764$ & $27 / 2122$ \\
\hline CDS (conserved genes/all): & $59 / 63$ & $81 / 94$ & $58 / 66$ & $26 / 46$ \\
\hline non-spliced (count/bases): & $61 / 38145$ & $93 / 69072$ & $26 / 15873$ & $29 / 15822$ \\
\hline spliced (count/bases): & 2/5 511 & $1 / 1467$ & $40 / 34449$ & $17 / 16299$ \\
\hline Introns (count/bases): & $2 / 1630$ & $1 / 2757$ & $160 / 55702$ & 61/NA \\
\hline Density (genes per kb): & 0,932 & 0,925 & 0,468 & 0,627 \\
\hline Average length (excl. introns): & 692 & 750 & 751 & 698 \\
\hline Coding percentage (excl. introns): & 64,5 & 69,4 & 35,1 & 43,7 \\
\hline Intergenic sequences (excl.RNA): & 12614 & 18720 & 25535 & NA \\
\hline Overlaping sequences: & 1161 & 1890 & 6209 & NA \\
\hline
\end{tabular}

group II introns often encode for reverse transcriptases, which probably help with their splicing and retroposition [27,28], the homology should be taken seriously. The second intron is much shorter (152 bp), without an ORF, and is located in the rpoB gene. The sizes of both introns (excluding ORFs) are smaller than typical group II and longer that group III introns, and we have not been able to find any noticeable similarities in the secondary structure with self-splicing introns in E. gracilis or elsewhere. Therefore, their ability to self-splice as well as their exact boundaries should be considered only putative. Besides the orf386 in intron 1, the Eutreptiella plastid genome encodes three other ORFs with homology to reverse transcriptases or intron maturases. Two of them (orf291 and orf372) have no close homologues, and their evolutionary origin cannot be traced. The third (matl) is clearly homologous to matl (ycf13) of E. gracilis and other euglenids, and in the tree (Figure S1) it forms a well supported branch (98\%) with these genes. Matl was apparently present in the last common ancestor of euglenid plastids but interestingly this reverse transcriptase is unrelated to the single reverse transcriptase found in the plastid genome of Pyramimonas (orf608) (Figure S1). Matl is also remarkably conservative regarding its position in the genome. In almost all investigated euglenids, including relatively closely related Eutreptia, it is situated in the internal group III intron of the $4^{\text {th }}$ intron in the psbC gene [18]. In Eutreptiella it is located right next to the psbC gene, which in Eutreptiella does not contain any intron. Matl was found also in the chloroplast of $E$. longa. As this organism has no psbC gene, the matl gene is situated in different loci [7]. The RT and X domains of E.gracilis and E.longa matl deviate from the consensus sequence of 34 group II intronencoded proteins [29]. Sequence alignment of matl in Eutreptiella and E.gracilis shows the loss of at least two conserved domains. Comparison between the genomes of E. gracilis, Eutreptiella and Pyramimonas suggests that the genome of the common ancestor of euglenid plastids was intron-poor but encoded at least one reverse transcriptase (matl). Expansion of introns is apparently a feature specific to E. gracilis and its relatives, as already suggested by Thompson et al. [17]. On the other hand, the small number of introns, their unusual sizes and structures and the loss of the otherwise-conserved intron in psbC indicate the suppression of introns in Eutreptiella. The evidence for the recent horizontal transfer of a group II intron from a cyanobacterial donor was found in the chloroplast genome of Euglena myxocylindracea [30]. This intron (in the psbA gene) includes ORF575, named mat4, which resembles cyanobacterial reverse transcriptases. Mat 4 is also homologous to the maturase of Pycnococcus provasolii and Volvox carteri (Figure $\mathrm{S1}$ ), which is located in an intron of the atpB gene [16].

The content of the unique protein coding genes is surprisingly similar between Euglena gracilis and Eutreptiella gymnastica plastid genomes (Figure 3). The Eutreptiella plastid encodes for the same photosynthetic proteins (31), transcription/translation proteins (5), ribosomal proteins (21), and maturase matl as Euglena gracilis. There are only 5 extra ORFs in Eutreptiella as compared with $E$. gracilis - four ORFs without strong similarity to known proteins (orf291, orf386, orf248 and orf372) and one conserved protein with homology to $P$. parkeae ycf65 (putative ribosomal protein rpl3). Similarly, only eight genes (including intron maturases mat2, roaA and orf506) are specific to E. gracilis. Not surprisingly, many of the shared proteins have been lost in Euglena longa, whose plastid has lost photosynthetic activity. Given this almost exact match of protein coding capacity of two genomes, whose last common ancestor was at the same time the last common ancestor of all known euglenid plastid genomes, we can with reasonable confidence expect that the Eutreptiella plastid genome also matches the coding capacity of this last common ancestor. Using the Pyramimonas parkeae plastid genome to represent the closest relative to the plastid endosymbiont, we may trace quite precisely the changes in the protein coding capacity of the plastid genome that took place right before and during the process of the secondary endosymbiogenesis. This coding capacity was reduced compared to Pyramimonas by the set of genes coding for: 10 proteins of NADH dehydrogenase complex, 2 proteins of cytochrome $\mathrm{B}_{6} \mathrm{~F}$ (petA, petN), 3 proteins of chlorophyll metabolism (ChlL, ChlN, ChlB), heme binding protein ccsA, photosystem I subunit psaI, initiation factor infA, the protease subunit of clp protease clpP, chloroplast division protein $\mathrm{FtsH}$, and several conserved and non-conserved ORFs with unknown function. A BLAST search of 23,372 transcriptome sequences of E. gracilis in GenBank and 268530 transcriptome sequences of Eutrepriella produced by us (unpublished data) revealed that transcripts for some of these proteins, 


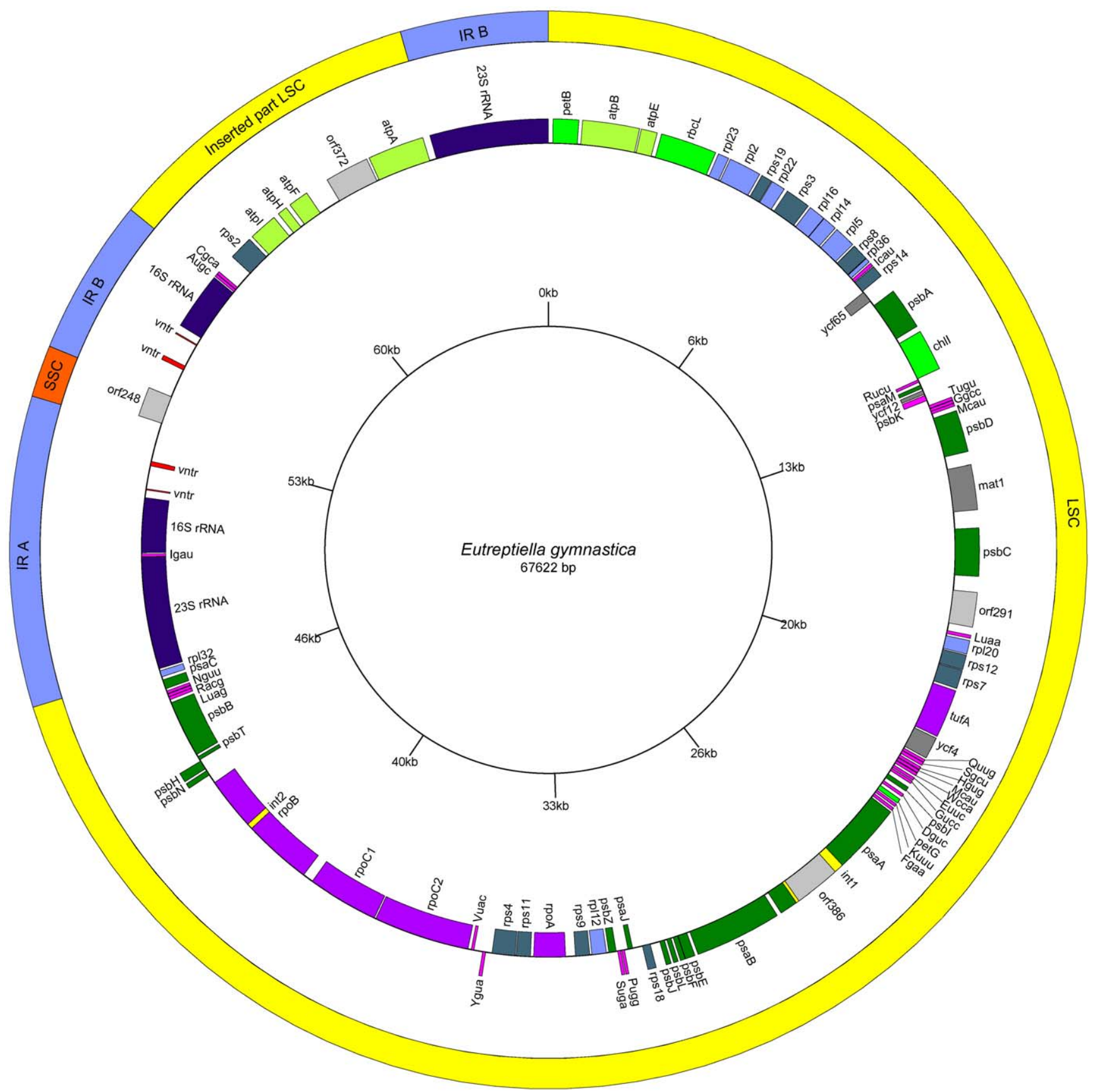

Figure 1. Map of the plastid genome of Eutreptiella gymnastica. Outer circle shows the large single copy region (LSC) (yellow), short single copy region (SSC) (red) and inverted repeats (IR) (blue). The inner circle shows genes and their division layout in respect to the DNA strands. The genes are color coded according to their function: photosynthesis (shades of green), translation (except maturases) (shades of blue), transcription (violet), tRNA (pink), maturases and unknown function (gray).

doi:10.1371/journal.pone.0033746.g001

namely petA, petN, ycf3, clpP, and ftsH, are present in both transcriptomes, indicating that these genes were probably transferred into the nucleus of the common ancestor of photosynthetic euglenids during the endosymbiogenesis. The gene ccsA is present only in the transcriptome of Euglena, suggesting that it was transferred into the nucleus of the common ancestor of photosynthetic euglenids, but retained in Euglena while probably lost in Eutreptiella. The rest of these genes were not found in any transcriptome. Although we cannot rule out the possibility that their transcripts were missed by transcriptome sequencing (e.g. due to the low abundance of transcripts), the observations here suggest that they might have been lost completely, either in the evolution of green algal ancestor of euglenid plastid after the split of the Pyramimonas branch, or later during endosymbiogenesis itself.

In contrast to the highly conserved gene content of E. gracilis and Eutreptiella gymnastica plastid genomes, the conservation of gene order is much lower between the two and also in comparison to Pyramimonas, indicating that many genome rearrangements have taken place. To get a rough estimate of the degree of gene conservation we counted the number of neighboring gene pairs 


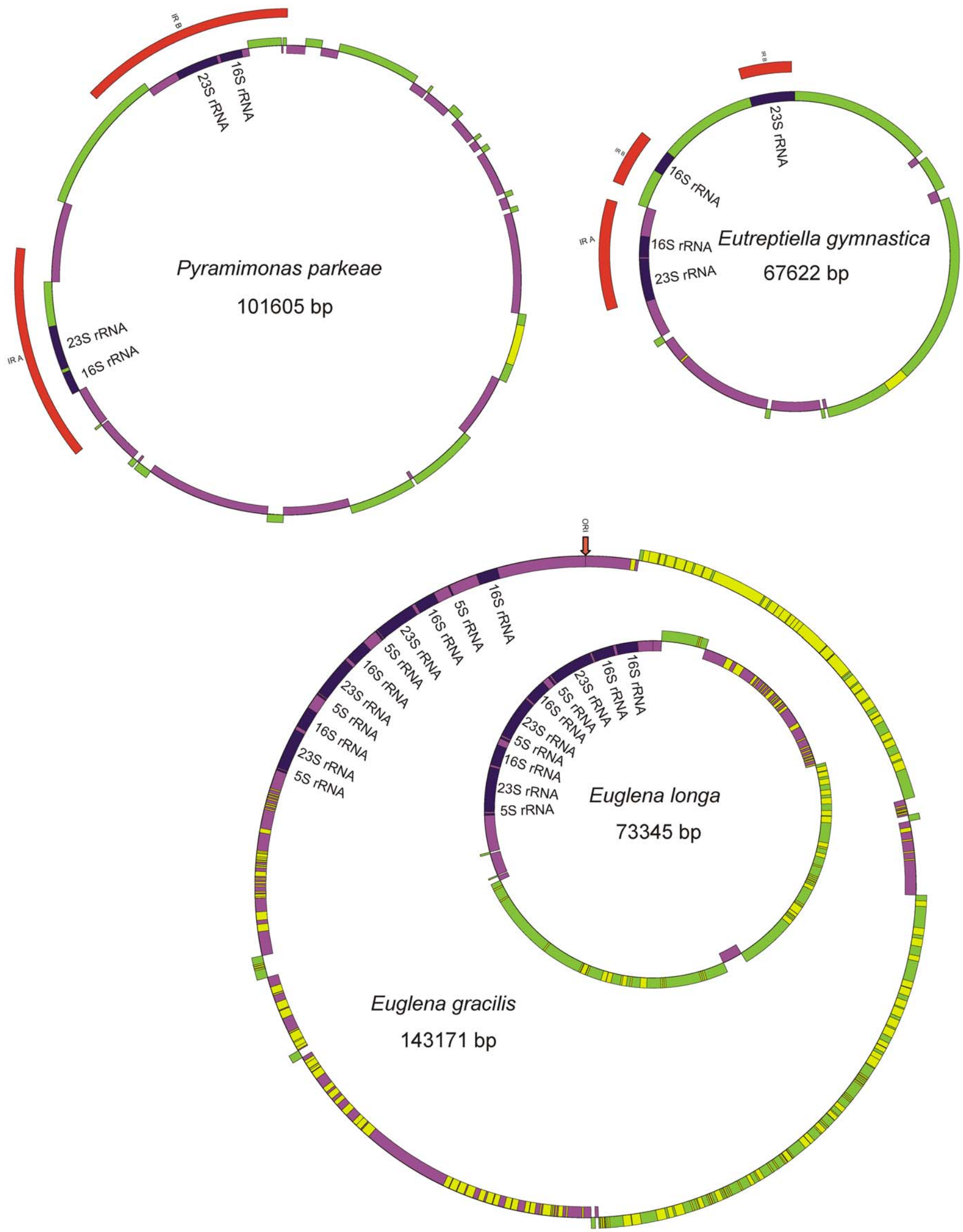

Figure 2. Simplified maps of the plastid genomes of Eutreptiella gymnastica, Euglena gracilis, Euglena longa and Pyramimonas parkeae. The maps are in scale to their sizes. The colors indicate the coding strands (plus-green and minus-violet), the ribosomal RNAs (blue) and introns (yellow). The inverted repeats IRA and IRB in Pyramimonas and Eutreptiella are marked in red. The ori site in Euglena gracilis is marked by an arrow. doi:10.1371/journal.pone.0033746.g002 
A

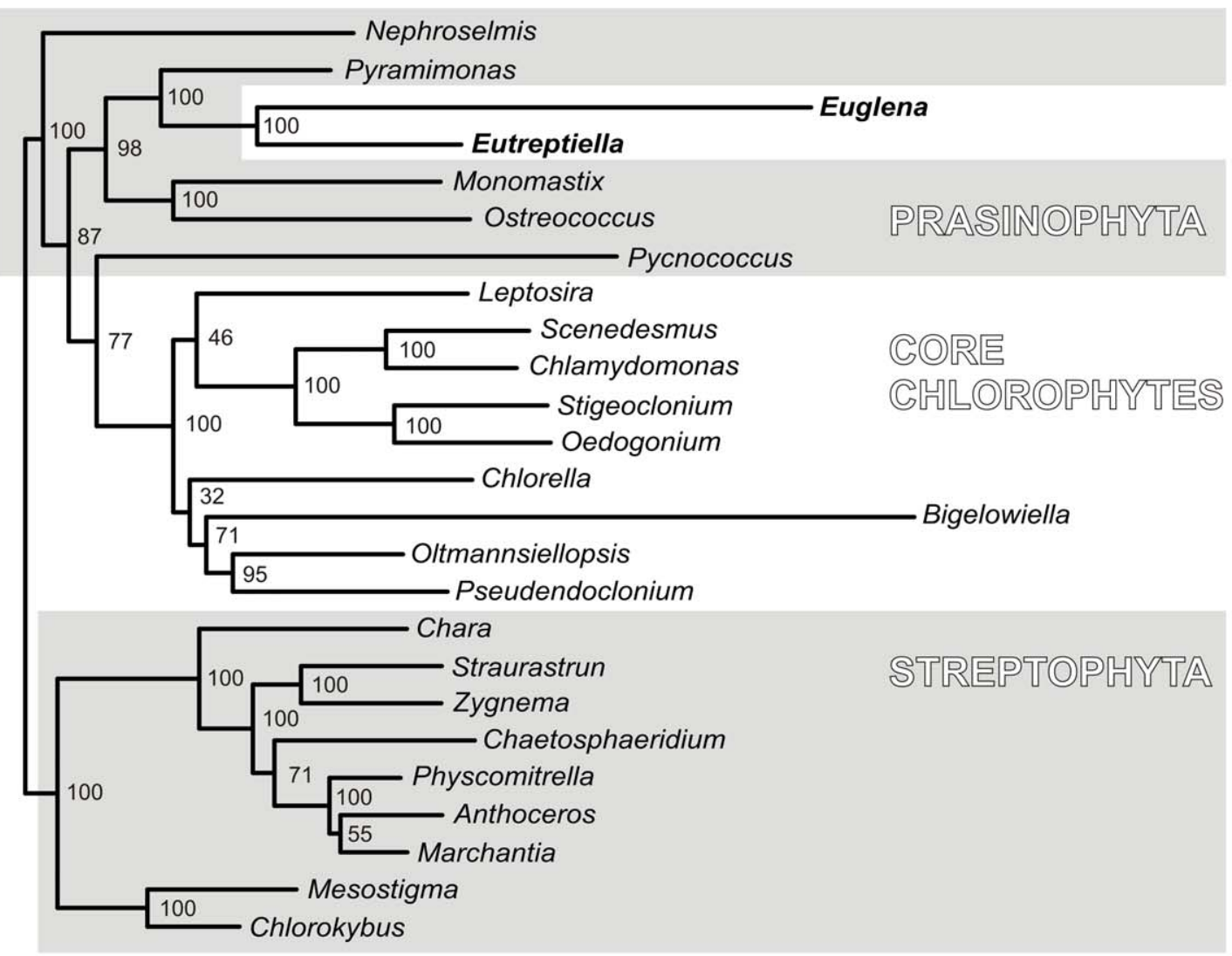

\section{1}

B



Figure 3. Venn diagrams showing overlaps in protein coding capacities between known euglenid plastid genomes and the plastid genome of Pyramimonas parkeae. The schematic representation of genome relationships is indicated in the left. Arrows indicate the probable fate of the genes absent from euglenid genomes. The genes are colour coded in respect to the functional group of their products: housekeeping proteins 
(black), proteins involved in photosynthesis (green), maturases of introns (red) and genes with unknown function (gray). Maturases of introns included in the phylogenetic tree of maturases (Figure S1) are marked by asterisks.

doi:10.1371/journal.pone.0033746.g003

common for pairs of genomes. In this measure, the E. gracilis and Eutreptiella genomes are the closest as expected, sharing 61 adjacent gene couples; Pyramimonas shares with each of them 40 and 38 gene neighbors, respectively.

Phylogenomic analysis of 70 plastid protein coding genes confirmed with maximum bootstrap support the sister relationship of euglenid plastids and Pyramimonas (Figure 4) as reported by Turmel et al. [16]. The tip branch of Euglena gracilis is almost three times longer than the branch of Eutreptiella, probably a result of an accelerated substitution rate in the lineage leading to the genus Euglena (Figure 4A). The analyses with relaxed molecular clocks produced ultrametric trees (Figure $4 \mathrm{~B}$ and Figure S2) that give estimates of relative ages of internal nodes. The branching order of these trees is virtually identical to the maximum likelihood tree. The relaxed clock analyses revealed that the common ancestor of Euglena and Eutreptiella (node 1 in Figure 4B and Figure S2) was not very recent, as it was approximately as old or older (depending on the clock model) as the common ancestor of vascular plants (common ancestor of Marchantia, Anthoceros and Physcomitrella). It also revealed that the age of the common ancestor of Pyramimonas and euglenid plastid (node 2 in Figure $4 \mathrm{~B}$ and Figure S2), for the three clock models, was 1.2-2.3 $\times$ older than the common ancestor of $E$. gracilis and Eutreptiella if considering the median of the age estimates and $1-5 \times$ older if considering the extreme values of the $95 \%$ confidence intervals of the age estimates (blue bars in Figures $4 \mathrm{~B}$ and Figure S2). The time span from node 2 to node 1 was therefore similarly as long as or shorter than the time span from node 1 to the present time, but likely was not markedly longer. The period from node 2 to node 1 includes the green algal lineage that became the direct ancestor of the secondary euglenid plastid and then the stem branch of the secondary plastid before the split of genera Euglena and Eutreptiella. The exact point where the transition between alga and plastid happened is not known. During this period, 16 protein coding genes functioning in the plastid metabolism were possibly lost and six were transferred to the nucleus of the euglenid. This is in contrast to the at least comparable but very probably quite longer time of evolution that separates extant photosynthetic E. gracilis and Eutreptiella (twice the time from node 1 to present) during which only one gene (ycf65) was lost and none was transferred to the nucleus. The rapid slowdown of gene loss could be explained by the fact that the gene set was relatively quickly reduced to an essential core that must be preserved if the photosynthetic function is to be retained. The complete halt of endosymbiotic gene transfer from plastid to the host nucleus is, however, unexpected, as such transfers are also reported in plastids that have been established for a long time in their hosts [31,32]. Unlike the gene content the gene order evolved relatively uniformly -61 gene couples remained in neighboring positions after the period separating $E$. gracilis and $E$. gymnastica, and correspondingly fewer (40 or 38) gene couples remained positionally fixed to each other after approximately double the period separating $P$. parkeae and $E$. gracilis or $P$. parkeae and $E$. gymnastica.

In conclusion, the plastid genome of Eutreptiella turned out to be almost identical to Euglena gracilis in protein coding gene content that is reduced when compared to Pyramimonas. This indicates that virtually all gene losses and endosymbiotic transfers of genes to the host nucleus took place in the period before the last common ancestor of the euglenid plastid. In contrast to the frozen protein content, the genome organization (gene order, inverted repeats) diversified significantly in the two sequenced lineages of euglenid plastids, and in the lineage leading to the genus Euglena it was furthermore accompanied by an accelerated substitutional rate in protein sequences and the expansion of self splicing introns. We have shown that the method of 454 sequencing could be widely applied to sequencing of organellar genomes.

\section{Materials and Methods}

\section{Preparation of genomic DNA}

A culture of Eutreptiella gymnastica strain SCCAP K-0333 was obtained from the Scandinavian Culture Collection of Algae and Protozoa and grown in TL30 medium in $12^{\circ} \mathrm{C} .150 \mathrm{ml}$ of wellgrown culture (approx. 25*10 $10^{6}$ cells) was used for DNA isolation. DNA was isolated using the Quiagen Blood and Tissue kit.

\section{Sequencing and assembly of the plastid genome}

$1 \mu \mathrm{g}$ of whole genomic DNA was subjected to 454 sequencing according to GS FLX Rapid Library Preparation Method protocol (Roche). In total 548056 reads of average size 370 bases were produced. Automatic assembly of reads in Newbler 2.5.3 (Roche) resulted in 19417 contigs (N50 contig size was 791 bases) and 9.2 $\mathrm{Mb}$ of unique sequence. Using a BLASTn homology search it was determined that two contigs, by far the longest (26 $365 \mathrm{bp}$ and $20813 \mathrm{bp}$ ), represented parts of the plastid genome. It is expected that contigs derived from the plastid genome should have approximately the same coverage, and so those contigs that had coverage similar to contigs 1 and $2(35 \times$ for contig 1 and $30 \times$ for contig 2) were selected from the assembly and all subjected to BLASTn homology search. Five of them were found to represent parts of the plastid genome. All plastid derived contigs were then manually assembled into a 67,274 bp long linear supercontig. Because we expected that the plastid genome would be a circular molecule, a PCR from the ends of the linear supercontig was used to amplify and sequence the missing part (primer F: 5' - taacctgtgaacacgaag $-3^{\prime}$ and primer R: 5' caaccagtaagttataggaa $\left.-3^{\prime}\right)$. After adding 348 bases the genome was circularized.

\section{Annotation}

Annotation of ORFs was done using BLASTx homology search. tRNAs were found using tRNA Scan-SE [33], and rRNAs were annotated using a BLASTn homology search with their boundaries determined according to the alignment with rRNA from Euglena gracilis and Pyramimonas parkeae. The annotation was completed in Artemis 13.2.0 [34] and the annotated genome is deposited in the EMBL database under accession no. HE605038. The genome maps were plotted in GenomeV [35].

\section{Intron secondary structures}

The secondary structures of intron candidates were predicted by mFOLD version 2.3 [36] (http://mfold.rna.albany.edu/ ?q = mfold/RNA-Folding-Form2.3) using the default setting but with the temperature set to $12^{\circ} \mathrm{C}$.

\section{Phylogenetic analyses}

The set of maturases was assembled from Eutreptiella matl and 121 homologues from GenBank representing both all available euglenid homologues and homologues from other taxa covering 
A
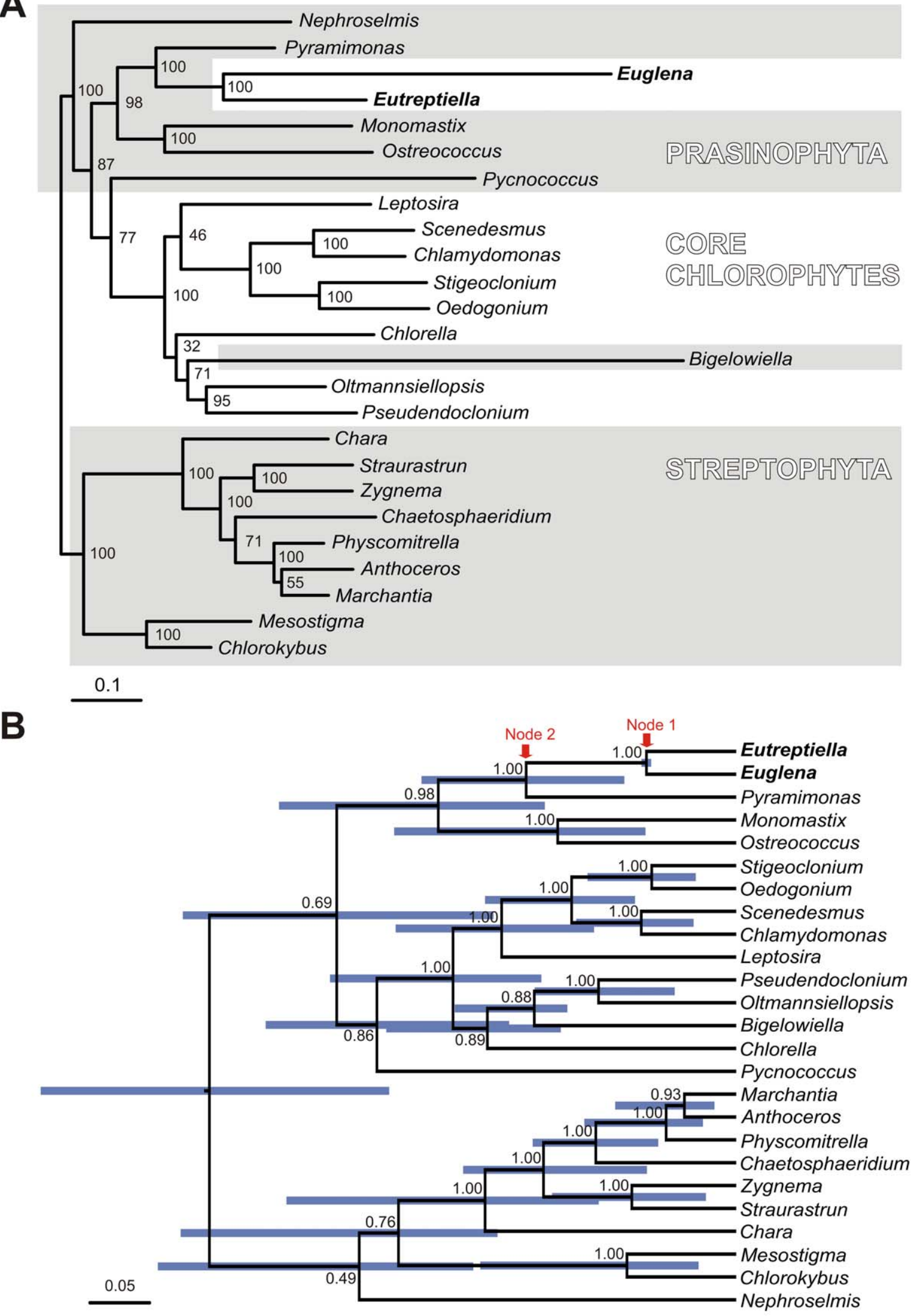

Figure 4. Phylogenies of plastid genomes of green algae, euglenids and Bigelowiella based on $\mathbf{7 0}$ genes. A. This phylogenetic tree was constructed using the maximum likelihood method implemented in RAxML, using the LG+I+G model selected by ProtTest. The bootstraps were estimated in 500 replicates. B. This tree was constructed in Beast v 1.6.1 using the WAG $+\mathrm{I}+\Gamma$ model of substitution and an uncorrelated exponential 
model of relaxed molecular clock. MCMCs were run for $10^{*} 10^{6}$ generations; trees from the first $2^{*} 10^{6}$ generations were discarded as the burn-in. Node labels represent posterior probabilities, node bars represent the $95 \%$ confidence interval of relative node ages.

doi:10.1371/journal.pone.0033746.g004

the sequential diversity of this protein. The data set was aligned using ClustalX [37] and manually edited in Bioedit 7.0.5.3 [38]. The phylogenetic tree was constructed in RAxML v7.2.7 [39] using the PROTGAMMAILGF model. The bootstrap support was calculated using the same model and 500 permutations.

For the phylogenomic analysis we used the data set of 70 protein coding genes from 24 plastid genomes published by Turmel et al [17]. The Eutreptiella sequences were manually added to this set in Bioedit 7.0.5.3 [38], realigned using ClustalX [37], and the alignment was then manually edited in Bioedit 7.0.5.3 [38]. The phylogenetic tree was constructed in RAxML v7.2.7 [39] using a uniform PROTGAMMAILGF model for all gene partitions. The bootstrap support was calculated using the same model and 500 permutations. The analyses using relaxed molecular clocks were performed in Beast v 1.6.1 [40] using the WAG $+\mathrm{I}+\Gamma$ model of substitution and three models of relaxed molecular clock: an uncorrelated exponential model, an uncorrelated lognormal model and a random model. MCMC was run for $10 * 10^{6}$ generations; trees from first $2 * 10^{6}, 7 * 10^{6}$ and $3 * 10^{6}$ generations were discarded as the burn-in, respectively.

\section{Supporting Information}

Figure S1 The phylogeny of intron maturases. The phylogenetic tree was constructed using the maximum likelihood method implemented in RAxML, using the $\mathrm{LG}+\mathrm{I}+\mathrm{G}$ model selected by ProtTest. The bootstraps were estimated in 500

\section{References}

1. Preisfeld A, Busse I, Klingberg M, Talke S, Ruppel HG (2001) Phylogenetic position and inter-relationships of the osmotrophic euglenids based on SSU rDNA data, with emphasis on the Rhabdomonadales (Euglenozoa). Int J Syst Evol Microbiol 51: 751-8.

2. Leander BS, Esson HJ, Breglia SA (2007) Macroevolution of complex cytoskeletal systems in euglenids. Bioessays 29: 987-1000.

3. Linton E, Hittner D, Levandowski CF, Auld T, Triemer RE (1999) A molecular study of euglenoid phylogeny using small subunit rDNA. J Eukaryot Microbiol 46: 217-223.

4. Marin B, Palm A, Klingberg M, Melkonian M (2003) Phylogeny and taxonomic revision of plastid-containing euglenophytes based on SSU rDNA sequence comparison and synapomorphic signatures in the SSU rRNA secondary structure. Protist 154: 99-145.

5. Marin B (2004) Origin and fate of chloroplasts in the euglenoida. Protist 155: $13-14$.

6. Hallick RB, Hong L, Drager RG, Favreau MR, Monfort A, et al. (1993) Complete sequence of Euglena gracilis chloroplast DNA. Nucleic Acids Res 21: $3537-44$.

7. Gockel G, Hachtel W (2000) Complete gene map of the plastid genome of the nonphotosynthetic euglenoid flagellate Astasia longa. Protist 151: 347-51.

8. Gibbs SP (1978) The chloroplasts of Euglena may have evolved from symbiotic green algae. Can J Bot 56: 2883-2889.

9. Leander BS, Triemer RE, Farmer MA (2001) Character evolution in heterotrophic euglenids. Eur J Protistol 37: 337-356.

10. Leander BS (2004) Did trypanosomatid parasites have photosynthetic ancestors? Trends Microbiol 12: 251-8.

11. Krajčovič J, Ebringer L, Schwartzbach SD (2002) Reversion of endosymbiosis? In Seckbach J, ed. Symbiosis: Mechanisms and Models. Cellular Origin in Extreme Habitats, Vol. 4. Dordrecht: Kluwer Academic Publisher. pp 185-206.

12. Vesteg M, Vacula R, Steiner JM, Mateášiková B, Löffelhardt W, et al. (2010) A possible role for short introns in the acquisition of stroma-targeting peptides in the flagellate Euglena gracilis. DNA Res 17: 223-231.

13. Hannaert V, Saavedra E, Duffieux F, Szikora JP, Rigden DJ, et al. (2003) Plantlike traits associated with metabolism of Trypanosoma parasites. Proc Natl Acad Sci USA 100: 1067-1071.

14. Bodył A, Mackiewicz P, Milanowski R (2010) Did trypanosomatid parasites contain an eukaryotic alga-derived plastid in their evolutionary past? J Parasitol 96: 465-75.

15. Maruyama S, Suzaki T, Weber APM, Archibald JM, Nozaki H (2011) Eukaryote-to-eukaryote gene transfer gives rise to genome mosaicism in euglenids. BMC Evolutionary Biology 11: 105. replicates. The eukaryotic maturases are marked by red, the cyanobacterial are marked by cyan and other bacterial maturases are marked by black.

(DOCX)

Figure S2 Phylogenies of plastid genomes of green algae, euglenids and Bigelowiella based on 70 genes. These trees were constructed in Beast v 1.6.1 using the $\mathrm{WAG}+\mathrm{I}+\Gamma$ model of substitution and an uncorrelated lognormal model of relaxed molecular clock (A) and random local model of relaxed molecular clock (B). MCMCs were run for $10^{*} 10^{6}$ generations; trees from the first $7 * 10^{6}$ and $3 * 10^{6}$ generations were discarded as the burn-in in A and B, respectively. Node labels represent posterior probabilities, node bars represent the 95\% confidence interval of relative node ages.

(DOCX)

\section{Acknowledgments}

The authors would like to thank to Dr. Steve Zimmerly for e-mail consultations regarding group II and group III introns and Aaron Heiss for language corrections.

\section{Author Contributions}

Conceived and designed the experiments: VH CV. Performed the experiments: JF JS SH. Analyzed the data: SH VH CV. Contributed reagents/materials/analysis tools: $\mathrm{CV} \mathrm{VH}$. Wrote the paper: $\mathrm{VH} \mathrm{SH}$.

16. Turmel M, Gagnon MC, O'Kelly CJ, Otis C, Lemieux C (2009) The chloroplast genomes of the green algae Pyramimonas, Monomastix, and Pycnococcus shed new light on the evolutionary history of prasinophytes and the origin of the secondary chloroplasts of euglenids. Mol Biol Evol 26: 631-48.

17. Thompson MD, Copertino DW, Thompson E, Favreau MR, Hallick RB (1996) Evidence for the late origin of introns in chloroplast genes from an evolutionary analysis of the genus Euglena. Nucleic Acids Res 23(23): 4745-52. Errata: Nucleic Acids Res 24: 542, 24: 1792, 1996.

18. Doetsch NA, Thompson MD, Hallick RB (1998) A maturase-encoding group III twintron is conserved in deeply rooted euglenoid species: are group III introns the chicken or the egg? Mol Biol Evol 15(1): 76-86.

19. Müllner AN, Angeler DG, Samuel R, Linton EW, Triemer RE (2001) Phylogenetic analysis of phagotrophic, photomorphic and osmotrophic euglenoids by using the nuclear 18S rDNA sequence. Int J Syst Evol Microbiol 51: 783-91.

20. Busse I, Preisfeld A (2003) Systematics of primary osmotrophic euglenids: a molecular approach to the phylogeny of Distigma and Astasia (Euglenozoa). Int J Syst Evol Microbiol 53: 617-24.

21. Geimer S, Belicova A, Legen J, Slavikova S, Herrmann RG, et al. (2009) Transcriptome analysis of the Euglena gracilis plastid chromosome. Current Genetics 55: 425-238.

22. Heinhorst S, Canon GC (1993) DNA replication in chloroplasts. J Cell Sci 104: $1-9$.

23. Bendich AJ (2004) Circular chloroplast chromosomes: the grand illusion. Plant Cell 16: 1661-6.

24. Ravel-Chapuis P, Heizmann P, Nigon V (1982) Electron microscopic localization of the replication origin of Euglena gracilis chloroplast DNA. Nature 300: 78-81.

25. Koller B, Delius H (1982) Origin of replication in chloroplast DNA of Euglena gracilis located close to the region of variable size. EMBO J 1: 995-8.

26. Morton BR (1999) Strand asymmetry and codon usage bias in the chloroplast genome of Euglena gracilis. Proc Natl Acad Sci U S A 96: 5123-8.

27. Michel F, Umesono K, Ozeki H (1989) Comparative and functional anatomy of group II catalytic introns-a review. Gene 82: 5-30.

28. Michel F, Ferat JL (1995) Structure and activities of group II introns. Annu Rev Biochem 64: 435-61.

29. Mohr G, Perlman PS, Lambowitz AM (1993) Evolutionary relationships among group II intron-encoded proteins and identification of a conserved domain that may be related to maturase function. Nucleic Acids Res 21: 4991-7.

30. Sheveleva EV, Hallick RB (2004) Recent horizontal transfer to a chloroplast genome. Nucleic Acids Res 32: 803-810. 
31. Huang CY, Ayliffe MA, Timmis JN (2003) Direct measurement of the transfer rate of chloroplast DNA into the nucleus. Nature 422: 72-6.

32. Bock R, Timmis JN (2008) Reconstructing evolution: gene transfer from plastids to the nucleus. Bioessays 30: 556-66.

33. Lowe TM, Eddy SR (1997) tRNAscan-SE: A program for improved detection of transfer RNA genes in genomic sequence. Nucl Acids Res 25: 955-964.

34. Rutherford K, Parkhill J, Crook J, Horsnell T, Rice P, et al. (2000) Artemis: sequence visualization and annotation. Bioinformatics 16: 944-5.

35. Conant GC, Wolfe KH (2008) GenomeVx: simple web-based creation of editable circular chromosome maps. Bioinformatics 24: 861-2.
36. Zuker M (2003) Mfold web server for nucleic acid folding and hybridization prediction. Nucleic Acids Res 31: 3406-3415.

37. Larkin MA, Blackshields G, Brown NP, Chenna R, McGettigan PA, et al. (2007) Clustal W and Clustal X version 2.0. Bioinformatics 23: 2947-2948.

38. Hall TA (1999) BioEdit: a user-friendly biological sequence alignment editor and analysis program for Windows 95/98/NT. Nucl Acids Symp Ser 41: 95-98.

39. Stamatakis A (2006) RAxML-VI-HPC: maximum likelihood-based phylogenetic analyses with thousands of taxa and mixed models. Bioinformatics 22: 2688-90.

40. Drummond AJ, Rambaut A (2007) BEAST: Bayesian evolutionary analysis by sampling trees. BMC Evol Biol 7: 214. 